\title{
EL ENFOQUE DE GÉNERO EN LOS CURSOS ACADÉMICOS DE LAS PRIMERAS MUJERES UNIVERSITARIAS BRASILEÑAS
}

\section{Carmem Silvia da Fonseca Kummer Liblik}

carmemsfk@gmail.com Universidade Federal do Paraná - Brasil

Recibido: 29-02-2016

Aceptado: 23-05-2016

\section{Resumen}

Gilda de Mello e Souza, Alice Canabrava, Olga Pantaleão, Jandyra Barzagui, Maria Conceição Vicente de Carvalho y Verônica Rapp de Eston forman un grupo de mujeres que cursaron la educación superior en la Universidade de São Paulo a finales de 1930, que consolidaron sus carreras como profesoras universitarias e investigadoras. Llamadas "pioneras" (las primeras mujeres que entraron en la Universidad brasileña), estas profesionales dejan en las declaraciones, escritas en la década de 80, la descripción de sus trayectorias académicas y profesionales impulsados por género. A través del análisis del discurso, se analizaron estos testimonios teniendo como guía dos eventos, a saber: la entrada de las mujeres en la educación superior y las dificultades iniciales que tenían para consolidar sus carreras en el nivel universitario de Brasil, entre las décadas de1940-1970.

Palabras Clave: Pioneras, trayectorias académicas, Universidade de São Paulo, género, discurso.

\begin{abstract}
Gilda de Mello e Souza, Alice Canabrava, Olga Pantaleão, Jandyra Barzagui, Maria Conceição Vicente de Carvalho y Verônica Rapp de Eston are part of a group of women who had higher education at the Universidade de São Paulo in the late 1930s, who have consolidated their careers as university teachers and researches. So-called "pioneers", these professionals left in the statements, written in the $80 \mathrm{~s}$, the description of their academic and professional trajectories driven by gender. Through discourse analysis, we analyzed these testimonies having as a guiding two events, namely: the entry of women in higher education and the initial difficulties they had to consolidate their careers in the Brazilian university level, between the decades of 1940-1970.
\end{abstract}

Keywords: Pioneers, academic trajectories, Universidade de São Paulo, gender, discourse. 


\section{Introducción}

La relación y la participación de las mujeres en el conocimiento científico e intelectual, y sus experiencias como estudiantes y profesoras en las universidades brasileñas, consisten en un tema relativamente reciente dentro de los estudios de género. Busca comprender cómo se establecen las relaciones de género entre alumnos, estudiantes, maestros, profesores y, sobre todo, las trayectorias de las mujeres cuando desean convertirse en un miembro del cuerpo administrativo y docente universitario; y/o de gran visibilidad nacional de investigadores (Nadia Lima, 2002; Marcia Tiburi, 2002; Eva Blay, 2006; Simere Santos, 2015; Carmem Liblik, 2015). También son comunes los análisis que reflejan cómo el género puede ser una categoría analítica importante que dio forma al conocimiento de algunas áreas científicas y profesionales. Además, salta a los ojos de las personas interesadas en este tema la presencia de las discusiones que tienen intención de conciliar la vida profesional y académica y la vida privada, como por ejemplo: el matrimonio, la familia y los hijos/niños.

En este contexto, las trayectorias académicas y profesionales de las primeras mujeres que se inscribieron en los cursos de la Facultad de Filosofía, Ciencias y Letras (de aquí en adelante FFCL) de la Universidade de São Paulo (de aquí en adelante USP), a mediados de la década de 1930, son objeto de estudio de este artículo, cuyos elementos se problematizan en el análisis del discurso. Para ello, elegimos los testimonios de las siguientes profesoras universitarias: la filósofa Gilda de Mello e Souza; la química Jandyra França Barzaghi; las historiadoras Alice Canabrava e Olga Pantaleão; la geógrafa Maria Vicente de Carvalho y la médica Verônica Rapp de Eston. Sus testimonios fueron escritos en la década de 1980, y revelan las experiencias que tuvieron en la universidad desde 1934, año en el que la primera, Jandyra França Barzaghi, ingresó en la educación superior. Una de las principales razones que llevan a la elección de estas profesionales se centra en el hecho de que eran las primeras mujeres que habían completado cursos de educación superior en la USP e hicieron sus trayectorias profesionales como profesoras e investigadoras universitarias.

Más que eso, siguieron a la creación e institucionalización de algunos cursos de la FFCL y, más tarde, fueron las principales representantes femeninas, las llamadas "pioneras", para el desarrollo de la investigación en relación con el postgrado. Vamos a analizar, por lo tanto, sus declaraciones en las que figuran descripciones de su infancia; entrada en la universidad y el contacto con los primeros materiales y maestros; la decisión de hacer un doctorado; concursos previstos para el puesto de profesoras y, finalmente, los caminos como investigadoras. A través de herramientas analíticas que nos ofrece el discurso, podemos ver las formas en que se expresan las dificultades que deben reconocerse con un espacio profesional que, en sus inicios, era predominantemente masculino. En las siguientes líneas, se describe una breve biografía y luego pasaremos a analizar sus discursos como un eje rector del mismo, es decir, los marcos de tiempo que los gobiernan. 
Olga Pantaleão nació en Ariranha, Estado de São Paulo, en 1917. Se graduó en Geografía e Historia por la FFCL/USP en 1938. En 1944 hizo su doctorado en Historia por la misma Universidad, con una tesis en Historia Económica. Entre 1939 a 1947 asumió el cargo de Primer Asistente y Profesor interino para la Historia de la Cátedra de Civilización Moderna y Contemporánea. Fue la fundadora del campus de la Universidade do Estado de São Paulo (de aquí en adelante UNESP) y profesora de Historia Moderna y Contemporánea como Titular de la Universidade de Filosofia, Ciências e Letras de Marilia, de 1959 a 1975.

Alice Piffer Canabrava nació en Araras, Estado de São Paulo en 1911. Al igual que Olga Pantaleão, licenciada en Geografía e Historia en 1938, en la FFCL. Doctorado en Historia en 1942, con una tesis en Historia Económica. Cuatro años después de obtener el título de doctor, se tornó Profesor Asociado de la Historia de la Civilización Americana de la FFCL y en 1951 obtuvo el título de profesor titular de Historia General y Brasil, en la Facultad de Economía y Administración de la USP, en la función hasta 1981. Alice también fue pionera en el desarrollo de la investigación dirigida a la Historia Cuantitativa e Historia Económica de la Universidade de São Paulo, además de ser una de los fundadores de la Associação dos Professores Universitários de História (de aquí en adelante ANPUH), así como de la Revista Brasileira de História (de aquí en adelante RBH).

La filósofa Gilda de Mello e Souza nació en São Paulo en 1919, y pasó gran parte de su infancia en Araraquara. Volvió a São Paulo en 1930 y se unió a la FFCL en 1937 graduándose en Filosofía en 1940. En 1942 fue Asistente del profesor de sociología Roger Bastide, impartiendo cursos relacionados con Estética, disciplina de la cual, más tarde, llegó a ser la responsable en el Departamento de Filosofía. En 1950, hizo el doctorado en Filosofía con una tesis titulada La moda en el siglo XIX. Prueba de la sociología estética. Fue Profesora-Jefe del Departamento de Filosofía entre 1960 y 1971 y fundó la revista Discurso, del Departamento de Filosofía, 1970-1976.

Jandyra França Barzaghi nació en 1915 en la ciudad de São Luiz de Paraitinga/SP. Ingresó a la USP en 1934, siendo alumna de la primera clase de la FFCL, admitida en Química de la Facultad de reciente creación. Antes de eso, se graduó en 1930 de la Escuela Normal de Pirassununga y en 1933 fue nombrada "preparadora de Física y Química" en la enseñanza primaria. En 1938, completó una graduación en la FFCL en Química y continuó sus estudios mientras hizo su doctorado bajo la dirección del profesor alemán Heinrich Hauptmann. En 1942, defendió su tesis Sobre Cafesterol y algunos de sus derivados y fue aprobada con distinción, cuando ya ocupaba el puesto de Asistente de Cátedra Orgánica y Química Biológica. En años posteriores fue una de los responsables de la organización del curso de Química de la USP y renunció en 1951, por razones personales.

La geógrafa Maria Carvalho Conceição Vicente nació en Santos, São Paulo, en 1906. Es licenciada en Geografía e Historia por la FFCL, en 1938. En 1944 defendió su Tesis en Geografía y en 1946 fue miembro del Consejo Nacional de Geografía, con sede en Rio de Janeiro en 1946. Fue becaria del gobierno francés en la Universidad de París, 1951-1952 y 
profesora de Geografía Humana de la Universidade Católica de Rio de Janeiro, de 1948 a 1958. Por último, fue Profesora Titular de Geografía en el curso de Historia de Filosofía, Ciencias y Letras de la Universidade de Julio de Mesquita Filho, Campus de Marília, 19591972.

Por último, se presenta Verónica Rapp de Eston, médica, nacida en São Paulo, en 1918. Se graduó en Medicina por la USP en 1944. Después de dos años de cursos, pasantías e investigaciones en los Estados Unidos y Canadá, con becas de estudio, se incorporó al Departamento de Química Fisiológica en la Facultad de Medicina de la USP. Fue fundadora y secretaria de la Sociedad Brasileña de Biología y Medicina Nuclear y la Asociación de Biología de América Latina y las Sociedades de Medicina Nuclear. Fue presidente de la Asociación Brasileña de Mujeres Médicas en el nivel regional y nacional, y se retiró en 1976 después de 30 años de servicio a la USP.

Cabe señalar que, si se trata de considerarlas como ganadoras, son pioneras aquí. Es cierto que hubo otras que llegaron a la misma posición e incluso aquellas que se han graduado, pero no siguieron la carrera docente en la universidad, o aquellas que han reorientado sus vidas siguiendo otras opciones. La selección de testimonios y la elección de estas profesionales se centran, como se explica en las siguientes líneas, en el contexto de la producción de sus discursos, escritos y comunicados en 1984.

\section{La primera temporalidad: las condiciones de producción de los estados y sus significados}

La teoría que apoya este texto se refiere al análisis del discurso de la línea francesa, que une la estructura lingüística a la vida social e histórica. En otras palabras, el lenguaje se estudia no sólo como forma lingüística, sino también como forma material de la ideología. Es "en contacto del material histórico con el lenguaje, que se obtiene la materialidad específica de expresión" (Michel Pêcheux, 2002:8). Entendemos que el discurso es la práctica social de la producción de textos, al ser una construcción social, no individual, y que sólo puede ser analizado teniendo en cuenta su contexto histórico y social y sus condiciones de producción. También significa que el discurso refleja una visión del mundo vinculado a los individuos y la sociedad en que viven.

Los testimonios analizados fueron escritos en julio de 1984, debido a la celebración del 50 aniversario de la fundación de la USP y la FFCL. Quien llevó a cabo todo el proceso de ponerse en contacto con antiguos alumnos, pidiendo sus declaraciones, transcribiéndolas y, por último, organizándolas en diferentes ediciones, fue la historiadora Eva Blay. Su propósito era reunir testimonios de los primeros estudiantes de la USP que luego después consolidaran su carrera académica en la educación superior como investigadoras y profesoras. Los testimonios se 
presentaron en 1984, cuando las pioneras tenían alrededor de 70 años de edad y se habían retirado hace unos pocos años de la universidad. Por último, la presentación de su experiencia como estudiantes y profesoras de la USP en la FFCL sucedió en la reunión del Sociedad Brasileña para el Progreso de la Ciencia (SBPC), en 1984. En este congreso, estas mujeres fueron llamadas "Las Pioneras". Algunas partes de los testimonios fueron publicados en un artículo escrito por Eva Blay y Alice Gordo Lang, titulado A mulher nos primeiros tempos da Universidade de São Paulo en la revista Ciência e Cultura, y después el material fue dejado de lado por varias razones. Después de 20 años, los informes, las informaciones de las entrevistas preliminares y algunas fotos fueron organizadas en un libro publicado por Eva Blay y Alice Lang, titulado Mulheres na USP: horizontes que se abrem.

El conjunto de narrativas memorialistas y autobiográficas viene a satisfacer la necesidad de las profesoras e investigadoras universitarias de expresar las experiencias del pasado, a menudo difíciles y polémicas, en el marco de la inauguración de los primeros cursos universitarios de la FFCL. Los testimonios están situados dentro de los límites del discurso, la autobiografía y la memoria. Son registros realizados en 1984 para llevar a través de la memoria, las experiencias que la universidad tenía como estudiantes y profesores a partir de mediados de la década de 1930, el momento de la creación de la FFCL. Debido a la finalidad de la historiadora Eva Blay para organizar entrevistas USP de sólo ex-alumnos que se han convertido en profesores e investigadores universitarios, la mayoría de los eventos tratan del proceso de incorporación de las mujeres en la universidad, la sociabilidad entre las jóvenes y los jóvenes de colegio, las relaciones que tenían las pioneras con sus profesores y las dificultades que tuvieron en los primeros años de la vida laboral. Pronto, sus discursos están específicamente alineadas con un eje central de la producción de contenidos mediada por la memoria: las experiencias de las mujeres en la universidad, ya sea como estudiantes, a veces como maestras.

Son discursos que se desvían del presente y se lanzan hacia el pasado. A través de varios criterios, los intelectuales en cuestión seleccionaran, organizaran y sistematizaran situaciones que fueron vivenciadas de acuerdo con el establecimiento de un objetivo común: traer las relaciones de género para el espacio universitario. Ellas politizan la universidad, los cursos y sus trayectorias profesionales trayendo el género como la clave que nortea la comprensión de este tipo de procesos.

Curiosamente, los testimonios de las pioneras están articulados en dos marcos de tiempo. El primero se refiere al período real de discursos que fueron escritos y presentados, es decir, a principios de 1980. Es precisamente en este período, o más bien a partir de la década de 1970, que los estudios de género, historia de las mujeres y las teorías feministas son recibidos por las investigadoras que trabajan en universidades brasileñas, en especial en la USP. A partir de entonces, tuvieron lugar los primeros estudios sobre la situación de la mujer en Brasil, sobre la base de los debates teóricos comenzados principalmente en Francia y Estados Unidos. Incluso teniendo en cuenta el hecho de que las profesoras universitarias no se denominaban feministas y 
no investigaban temas relacionados al género o con las "mujeres", que no eran ajenas a este proceso.

Las universitarias en cuestión se insertan en una institución que comenzó a recibir y producir las primeras obras del área y, por lo tanto, directa o indirectamente, socializaron con las investigadoras feministas y transitaron en los mismos espacios que ellas. A partir de sus experiencias, a continuación, mediadas por los debates feministas que entraron en las universidades públicas del país a principios de 1970, es que la memoria de ellas pudo construir sentido y dibujar interpretaciones sobre las experiencias pretéritas. Apenas se darían cuenta y configurado de la misma manera sus experiencias si las hubieran narrado en la década de 1930, en 1940 o 1950. Así es que podemos entender la constitución de significados inherentes en el testimonio de las pioneras en cuestión, es decir, el reconocimiento de las condiciones de producción de su discurso". Como escrito por Eni Orlandi, "hablar en discurso es hablar en condiciones de producción", en las cuales están los sitios sociales de interlocutores y su posición relativa en el discurso (Eni Orlandi, 1983: 146).

Las primeras páginas del testimonio de Alice Canabrava, por ejemplo, se refieren al momento histórico en que ella escribió, que jugaran un papel decisivo en la elección de los temas tratados en el texto, especialmente aquellos articulados por las dificultades que tuvo en la vida laboral como una mujer. Ella desarrolla inicialmente nociones de una sociedad prejuiciosa con las mujeres, en los que en "su mayoría [ellas] se mantienen en la historia como figuras silenciosas, en gran parte desconocidas, madres, esposas o hijas sin su propia voz". La historiadora recuerda a la mujer como "figura silenciosa," impedida durante mucho tiempo de comunicar su propia visión del mundo, de modo que los testimonios de las mujeres son relativamente "poco frecuentes en la literatura histórica brasileña". Se hace hincapié en que sólo en los últimos años se han producido iniciativas que buscan "rescatar" la memoria femenina.

Sin embargo, ella no pretende generalizar y decir que todos los hombres se comportaban de la misma manera con el sexo opuesto en el ámbito universitario. Para la historiadora, aunque no se diga, "grandes personas se encuentran en el interior de su tiempo y de sus medios". Entonces ella tiene el conocimiento para distinguir a las personas que se oponían a su carrera a de los que no tienen un comportamiento hostil. Tampoco lo hace como una víctima singular que enfrentó, sin ayuda, las dificultades impuestas, ya que ella percibe la discriminación que afecta a las vidas de otras mujeres de la universidad. Las primeras líneas de su testimonio se dedican, en primer lugar, aclarar que no mantuvo ningún resentimiento a los que eran hostiles a ella, y en segundo lugar, para situar el contexto histórico y social inherente a su carrera profesional:

"No he salvado resentimiento en relación con los que se oponían a mi subida en la Universidad. Las grandes personas estaban dentro de su tiempo y de sus medios, exponentes de una sociedad con prejuicios hacia las mujeres. No penséis que he sido el único objeto de

\footnotetext{
1 "Las condiciones de producción se definen a partir de las relaciones entre los lugares que los sujetos se atribuyen mutuamente cada uno a sí mismo y al otro" (Hall, 2010).
} 
discriminación: esto estaba dirigido a todos. En mi caso, mi resistencia causó el curso del proceso. En otros, esto se interrumpe o no fue efectuado, dado el alejarse del interesado, sea voluntario, sea por imposición de las circunstancias" (Alice Canabrava, 2003: 27).

Del mismo modo, Olga Pantaleão también articula sus recuerdos con las dificultades que encuentran al comienzo de su vida profesional. A pesar de haber sido contratado como Primero Asistente de la Historia General, indicada por el profesor Jean Gagé, que siempre le dio "pleno apoyo", ella cuenta que:

"Entonces las cosas empezaron a cambiar. Una reacción violenta masculina contra la mujer comenzó a manifestarse debido a varios factores: cuando la mujer mostraba ser más competente que los hombres en el mismo campo de estudio y la enseñanza, cuando ella ocupaba o pasa a ocupar lugar deseado para un colega de sexo masculino o simplemente debido a los prejuicios antifeministas” (Olga Pantaleão apud Eva Blay, 2004: 112).

Como Alice Canabrava, Olga Pantaleão no especifica cómo se trataba la "reacción violenta masculina contra las mujeres”. ¿Sería una reacción por parte de los compañeros de clase? ¿O serían, sobre todo, los profesores catedráticos quienes no deseaban compartir su cátedra con las mujeres jóvenes recién formadas? No se puede saber con exactitud qué tipo de hostilidad sufrieron, ni cuanto impactó y de qué forma en sus carreras. Sin embargo, lo que llama la atención en la habla de Olga es el uso del término "anti-feminismo.” ¿Era posible que los hombres de su tiempo fuesen comprendidos a partir de sus actitudes antifeministas? Creemos que no, simplemente porque los estudiantes en ese momento no eran feministas y porque la "acción masculina" no se direccionaba a una lucha feminista.

Aquí se ve algo interesante que puede ser entendido a la luz de la intertextualidad (Diana Barros y José Fiorin, 1999). Olga, cuando escribió su testimonio en el comienzo de la década de 1980, pasó por varias generaciones de profesores e investigadores, asistió a reuniones destinadas a la formación de una asociación de mujeres universitarias, e incluso narró sus experiencias articuladas a un hilo en particular: la "lucha de las pioneras ". Así que se aplica el concepto de "reacción antifeminista", recientemente adquirido en su momento como un modelo para entender y dar sentido a la conducta masculina pasada.

El hecho es también que Olga Pantaleão produce el lenguaje y también se reprodujo en ella, creyendo que es la fuente exclusiva de su discurso cuando, de hecho, su voz procede también de otros discursos. En este caso, corresponde reconocer la gama completa de los testimonios que se dieron por mujeres que entraron en la USP en el 1930 y que allí hicieron sus carreras académicas. Sus palabras, por lo tanto, son parte de las formaciones discursivas. Después de todo, según lo declarado por Eni Orlandi, "las formaciones discursivas determinan qué se puede y se debe decir de una posición dada en una situación dada, por lo que es que consideramos el discurso como un fenómeno social" (Eni Orlandi, 1983: 146). 
Alice Canabrava nos informa al principio que dejó "pasar los dedos en la máquina de escribir, al toque ligero de reminiscencias" y que no "omitió algunos detalles que le parecían importantes para caracterizar el medio ambiente.” Paradójicamente, en la misma frase que deja claro que otras informaciones también importantes, las dejaría para un posible libro autobiográfico, que en realidad nunca fue escrito. Estos pasajes son propensos a pensar en lo que dijo Michel Foucault acerca de la interdicción de la palabra, es decir, de qué manera pensamos acerca de la definición de lo que puede ser dicho o no dicho, dependiendo de cada circunstancia. En la interdicción, "no tenemos derecho a decir lo que sentimos, que no podemos hablar de cualquier cosa en cualquier circunstancia, cualquier persona puede no hablar de lo que quiere que sea" (Michel Foucault, 1998: 7). Alice Canabrava es una historiadora que era consciente de que su declaración podría ser leída por un público determinado: los historiadores, ya sean estudiantes o profesores. Son sus colegas profesionales, con los cuales, cuando ella incluso se retiró, mantuvo las relaciones y redes de contacto. Además, se debe tener en cuenta los intereses de todo intelectual, independientemente de la zona que actúa, para preservar una imagen pública sólida y respetable por sus compañeros de trabajo.

Por lo tanto, no todo lo que se puede hablar, expone y se revela. Sin embargo, para Foucault, se sabe que "las regiones en las que la malla está más apretada, en la que se multiplican los compartimentos negros, son las regiones de la sexualidad y las de la política" (Michel Foucault, 1998: 8). Hablando de Alice Canabrava aparentemente puede incluso "no ser nada" - como dice Foucault, sin embargo, prohibiciones que afectan el habla, revelan inmediatamente su relación con eventos que pueden referirse a los colegas, las situaciones de conflicto en el entorno profesional, disputas, por último, el afecto y el malestar en el espacio universitario. Un ejemplo de esto se ve cuando ella relata las dificultades que se le presentaban al principio de su vida profesional. Al finalizar su tesis doctoral titulada "El comercio portugués en el Río de la Plata," Alice Canabrava dice que "recibió elogios por la crítica nacional y extranjera, publicados en revistas internacionales." De hecho, sin detallar las indicaciones o por otros acontecimientos cuyos "personajes masculinos" eran hostiles, sino sólo que la relación con sus colegas masculinos cambió después de haber recibido elogios por su tesis:

"De este modo, sin darse cuenta, surgió en los ojos de mis colegas masculinos como posible candidata para la provisión efectiva de la cátedra universitaria de Historia Americana, a ser puesta en concurso. Hasta entonces, la relación con estos compañeros había sido muy afable, diría hasta que, no sin estima personal. Desde entonces, el círculo subterráneo de hostilidad comenzó a aparecer a mí con pruebas, para apretar de forma anónima en cualquier ocurrencia de la actividad universitaria sin cambiar la apariencia de cordialidad superficial" (Alice Canabrava, 2003: 19).

Las pioneras se colocan en el papel de los agentes que vuelven a contar sus experiencias del pasado. Esta es la forma en que construyen el paisaje de su autoridad enunciativa. Para ser enunciada, establecen una especie de "lenguaje ritual social", que en este caso es el lenguaje 
inherente en el campo del trabajo del historiador. En este sentido, Dominique Maingueneau nos da una clave para entender el sujeto que habla y la posición que ocupan los pioneros en relación con sus socios: el enunciado producido por un sujeto se coloca en contacto acreditando su lugar titular del conocimiento. Ellas tienen la legitimidad de su lugar en este proceso de la enunciación, y sus enunciados son estudiantes o profesores universitarios (Dominique Maingueneau, 1989: 30). Por extensión, las profesoras de la universidad en la definición de diversos momentos de texto, como veremos, su "lugar de la verdad", lo que significa que insertar la información que las legitiman como profesionales influyentes que toman el lugar del conocimiento.

\section{La segunda temporalidad: reflexiones sobre un pasado no tan lejano así.}

La segunda temporalidad presente en los testimonios se refiere al establecimiento de un devenir marcado por la vida, en particular la vinculada a las experiencias académicas. En éstas, la preocupación de las maestras seria narrar los acontecimientos que eran más importantes, siguiendo un orden cronológico y lineal. Este tiempo comienza en la infancia, en el caso de Gilda de Mello e Souza, Jandyra França Barzaghi, Alice Canabrava, y termina en los logros profesionales, por todas ellas. En este sentido, las pioneras cuentan sus historias de vida, tejiendo los hilos de recuerdos, que son mezclados, organizados y sumados de manera que un evento, una persona o un afecto llevan a la memoria de otros eventos que fueron importantes.

Si Olga Pantaleão, Maria Vicente de Carvalho y Verônica Rapp de Eston no comparten sus historias familiares, porque desde el inicio de su testimonio sólo escriben a partir del momento en que entraron en la FFCL, las otras pioneras no niegan informaciones sobre sus historias personales de niños en cuanto estaban en el interior de São Paulo. El efecto causado por este dispositivo de narrativa es claro: para expresar la entrada en la FFCL fue un punto de inflexión en sus vidas. Con esta división, es evidente que hubo un "antes", durante el cual vivieron como todas las mujeres de ese tiempo vivido, es decir, inmerso en un entorno tradicional, religioso y patriarcal, a pesar de sus tensiones con la presencia de nuevos modelos de conducta femenina; y “después", cuando marcado como una inmersión en nuevos horizontes, especialmente el cultural y profesional, que la universidad promueve la vida de estas mujeres.

Pasando gran parte de su infancia en Araraquara, Gilda de Mello e Souza fue inscrita en el curso de Filosofía en 1937 a los 17 años de edad. Su educación se limitaba al gimnasio y el segundo año de preuniversitario, que tuvo que asistir a la Facultad de Derecho. Señala que desde los 12 años vivía en un entorno, "generoso y hospitalario que, desde el siglo pasado, servía como un enlace entre el país y la capital y que albergaba las generaciones sucesivas de familiares más rústicos en la enseñanza y educación de la demanda." Gilda de Mello e Souza describe el entorno familiar en el que creció para ser sui generis: por un lado, estaban los 
hábitos provinciales mantenidos por los más viejos; por el otro, había la presencia de familiares que traían nuevos libros para la biblioteca, los cuales fueron decisivos para su formación:

"Por esta razón, yo ya era, a los 18, una chica inquieta, minada por la diferencia entre el viejo y lo nuevo, dividida entre el estilo patriarcal que reinaba en la casa, y el nuevo atractivo de la vida que la biblioteca estaba proponiendo. Las conversaciones dispersas con mi primo entre las clases de piano; el análisis de mis primeros intentos literarios, que el se comprometía con cuidado impresionante; la discusión de lo que yo quería ser en la vida - todo madurado en nosotros con la convicción de que yo debería inscribirme en la universidad, de preferencia en el curso de Filosofía" (Gilda e Souza apud Eva Blay, 2004: 65).

Alice Canabrava, a su vez, nació en Araras y en esta ciudad se dedicó durante cuatro años a la escuela primaria, enseñando en la escuela local, la única del municipio. Ella trabajó también en 1931 en Manduri, un "pueblo en medio de un gran campo de arena", donde sólo había una calle principal, pero no el cine, médico o plaza que podría referirse a la estructura de un centro urbano. Y, por último, cuando "transferida a Araras, mi ciudad natal, la encontré estancada como había conocido en mi infancia”. Era una pequeña ciudad, desgarrada por luchas políticas", lo que dificultaba progresos profesionales o académicos para la joven estudiante. Sin muchas perspectivas de crecimiento personal y profesional, e interiormente "siempre insatisfecha”, Alice Canabrava diariamente hojeaba las páginas del Diário Oficial, en busca de una oportunidad para trasladarse a São Paulo y seguir estudiando.

Posiblemente, esta información se proporciona para cumplir un objetivo discursivo: la intención de mostrar el entorno familiar en el que se encontraban las pioneras, lejos de la capital del estado, absorbidas en un trabajo muy diferente al que en el futuro irían elegir. En esta misma dirección, Alice Canabrava llama la atención sobre el hecho de que estas memorias pueden parecer muy alejadas de la finalidad que reunió a los pioneros en la reunión de la SBPC en 1983. Sin embargo, como ella dice, este tipo de memorias "no significan narrativa sin objetivo porque la intención era mostrar el "ambiente en el que se movía, lejos de la capital del estado, muy diferente del espacio de trabajo que elegiría para ir a la universidad".

Para entender las razones que llevaran Alice Canabrava, Gilda de Mello e Souza y Jandyra Barzaghi a presentar los recuerdos de su infancia y adolescencia, la experta del análisis del discurso y crítica cultural, Leonor Arfuch, saca a la luz el tema de la genealogía en el interior de las biografías. Es decir, ningún estado de situación viene "gratis" en el espacio discursivo de la expresión de los que tejen su propia biografía: el reto es encontrar una voz autobiográfica que puede dar sentido a un mito de origen, una genealogía, a un devenir predestinado. Por lo tanto, el espacio biográfico - la narración de historias y experiencias, captación de las vivencias y recuerdos - opera completamente en este "rescate" del mismo lugar de origen (Leonor Arfuch, 2010: 60).

En la misma dirección, Benito Schmidt también llama la atención sobre el hecho de que en muchos trabajos biográficos, se puede ver que los autores buscan en la infancia y/o 
adolescencia una especie de predestinación para sus actividades futuras (Benito Schmidt, 2000: 58). En otras palabras, la experiencia en São Paulo y sus limitaciones, la presencia de un "espíritu curioso e inquieto," la tensión que enfrentaron coexistiendo entre lo "viejo" y "nuevo" están relacionados con las razones que impulsaron estas mujeres para ir a la capital del estado y decidirse por una graduación. La falta de oportunidades de empleo, el estímulo y el crecimiento de estas ciudades les causó graves problemas que deben superarse mediante la educación y el trabajo. Por lo tanto, no es el principio de todas las declaraciones, informes sobre las limitaciones impuestas a las mujeres en el trabajo y la opresión de la sociedad patriarcal que las impedía de circular en las zonas comunes, como se ha señalado por la geógrafa Maria Vicente de Carvalho:

"En una sociedad patriarcal, como São Paulo y luego, muy parcial, ya que el trabajo de la mujer fuera del hogar, la educación primaria era el campo casi exclusivo de trabajo que se les ofrecía. Pocos se atrevieron a aventurarse fuera de ellos, haciendo frente a inscribirse en la escuela de derecho, y menos aún en la medicina o la ingeniería. Y, aunque éstas no siempre siguieron la profesión” (Maria de Carvalho apud Eva Blay, 2004:119).

Otra cuestión que se aborda en un testimonio aquí tratado que se refiere al creciente número de mujeres que ingresan en los cursos de la FFCL, especialmente de Geografía e Historia. En este sentido, ¿podemos decir que hubo un proceso de feminización en este curso de la década de 1930? La respuesta es definitivamente positiva teniendo en cuenta el amplio acceso y el estímulo que las mujeres recibieron del gobierno del país para unirse a nosotros en los cursos de la FFCL. La necesidad de que la preparación de los docentes para la educación secundaria se está expandiendo en el país, llevó a los gobiernos estatales a utilizar, a partir de 1935, al comissionamento a través del cual los maestros y profesores de escuelas primarias en el ejercicio de enseñanza podrían asistir a la universidad sin perjuicio de sus salarios, subvencionados por el Departamento de Educación (Eva Blay, 2004: 51). Esta fue la primera política pública implementada en la Universidade de São Paulo, un año después de su creación.

Este proceso fue uno de los factores determinantes para la inclusión de los alumnos y estudiantes de diferentes condiciones socioeconómicas de los distintos grupos de edad, las mujeres y los hombres, portadores de diversas graduaciones. El alumnado, a partir del segundo año de funcionamiento de la FFCL/USP, comenzó a incluir la clase media, la gente del interior del estado, las familias vinculadas a la enseñanza, las familias empobrecidas tradicionales, y en especial las mujeres (Maria Helena Trigo, 1997: 68-9, 77). A pesar de que la política tenía como principal objetivo la formación de profesionales para la enseñanza primaria y secundaria, esta acción tuvo otra consecuencia, no planificada previamente: sistema de comissionamento ha permitido una importante presencia de las mujeres en la FFCL, ya que representaban la mayoría de los graduados las escuelas normales del Estado. Una nueva realidad para el momento en que era inusual la presencia de las mujeres en la educación superior en Brasil. 
Esta afirmación se hace más evidente cuando se mira en la sección de Geografía e Historia, que no contaba con ninguna mujer entre los 16 registrados en 1934. Sin embargo, según el Anuario de la FFCL en 1935, el año en que el decreto es eficaz, hay 16 inscripciones femeninas, con un total de 29 en los años siguientes, el número de mujeres sigue superando el número de hombres. La sección de Filosofía también refleja esta realidad: hay 12 matrículas femeninas en 1935, de un total de 27, contra la única mujer inscrita en un total de 46 estudiantes inscritos en el año anterior (Eva Blay, 2004: 53). Fue a través del comissionamento de las pioneras que se logró entrar en el FFCL, cuyo hecho es especialmente destacado en sus declaraciones y en relación con ciertas dificultades y prejuicios a las mujeres:

"La Facultad desde su segunda clase, fue invadida por las mujeres en casi todos sus cursos, lo que constituye un caso especial en toda la Universidad: otras escuelas recibieron muy pocas mujeres y algunas de ellas todavía estaban cerradas, principalmente porque todavía existían prejuicios en la sociedad. Esta entrada de las mujeres en la filosofía fue permitida por varios factores: la apertura de vestibular a los graduados de las escuelas normales, las instalaciones, tales como comissionamento, dado a maestros de primaria para hacer los cursos" (Olga Pantaleão apud Eva Blay, 2004: 109).

Este decreto, además de promover una mejora en la primaria estatal, también tuvo una consecuencia prevista por los autores de medida: la posibilidad de un camino para la entrada de las mujeres en la universidad pública. Abierta esta posibilidad, muchos de ellas decidieron seguir una carrera académica, refutar cualquier idea o iniciativa de trabajar en la enseñanza primaria o secundaria. La FFCL permitió a las pioneras una oportunidad amplia y real de entrada en la educación superior. Como afirma Jandyra Barzagui: "Por supuesto, no había duda de que las mujeres sólo podían desean estudiar en la Escuela Normal, y las pocos que lo hicieron, eran pioneras que arduamente consiguieron su lugar entre los hombres" (Jandyra Barzagui apud Eva Blay, 2004: 78). Abríase un espacio y una oportunidad para nuevos caminos, especialmente una vida intelectual, en medio de una sociedad que abogaba por un modelo tradicional, conservador para las mujeres: el matrimonio, el hogar y los niños.

Al ingresar a la FFCL en 1935 para asistir a los cursos de Geografía e Historia, Alice Canabrava sentía "incómodo ante la grandeza de los profesores de la institución, pero profundamente entusiasmada" (Alice Canabrava apud Eva Blay, 2004: 91). Los maestros, la metodología y el contenido del curso de enseñanza destinados para ella significaran una "transformación real" en su vida. Ellos representan un gran salto en el tipo de educación que había recibido en la Escuela Normal, ya que la universidad le permitiría "reflejar la vida cultural del país y proporcionar una visión de todo el mundo nuevo" (Ibídem., 2004: 92). Es curioso observar que los demás deponentes utilizan términos y expresiones similares para referirse a la primera impresión que tenían cuando entraron en la USP. Gilda de Mello e Souza señala que ella era uno de los estudiantes más jóvenes de su clase y que dio la bienvenida "con entusiasmo a esta extraordinaria aventura que me lanzó, desde el primer día a la noche, la excitante 
atmósfera intelectual de una universidad típicamente europea" (Gilda e Souza apud Eva Blay, 2004: 63). Jandyra Barzagui, a su vez, dice lo siguiente:

“[...] Los maestros maravillosos que se asignaron! Conferencias, clases brillantes experimentales, que se abrían para el conocimiento ilimitado, verdadera pasión, que miraban durante más de dos horas sin darse cuenta de que nos fuimos con la vergüenza, que ansiaban escucharlo; el primer contacto con la investigación científica, que no escatimó esfuerzos ni contar las horas, que la dedicación y el esfuerzo más allá de las fuerzas que llevaron mi saudosismo Prof. El Dr. Heinrich Hauptmann como la muerte prematura; el día completo de clases prácticas, en las que permanecieron en estrecha comunión con los maestros y compañeros. Eran aquellos tiempos mágicos" (Jandyra Barzagui apud Eva Blay, 2004: 82).

"Tiempos mágicos", "aventura extraordinaria", "emocionante atmósfera intelectual” son expresiones que causan un efecto significativo de sentido. No sólo las pioneras, pero podemos decir que casi todos los estudiantes de la USP expresaron su profundo entusiasmo y aprecio por el contacto que tuvieron con un nuevo modelo educativo expuestos en las clases de los maestros europeos. Era, para ellos y ellas, una revolución en la educación en comparación con lo que habían aprendido en la escuela Normal, Clásico o Científico. La innovación no fue sólo el tipo de clases que se le dio, pero como se ha dicho por Eva Blay, "el contenido y la relación que se establece entre los profesores y estudiantes" (Eva Blay, 2004: 10). Una nueva y diferente sociabilidad estaba siendo experimentada por estas mujeres que hasta entonces tenían sus círculos de sociabilidad restringida a la familia.

El espacio universitario que estaba siendo creado en la década de 1930 tenía la característica de que afecta a distintos elementos en relación a las normas vigentes de espacio y la sociabilidad de las veces. Para el/la alumno(a), este proceso significó ser insertado, según lo informado por Graziela Perosa, en un espacio público, mezclado, fuera del control moral restringido, impuesto por la religión y las familias en las que las mujeres están expuestas a la influencia de una ambiente intelectual, secular y la convivencia con personas de diferentes orígenes sociales y culturales (Graziela Perosa, 2005). El intercambio y las amistades consolidadas entre las estudiantes y los estudiantes son recordados por Gilda de Mello e Souza de la siguiente manera:

"El nuevo vivir [...] En otra ocasión se me permite hablar más en profundidad en la atmósfera de la fascinación ejercida sobre nosotros por los profesores, que, fuera de las clases, continuaban las conversaciones alumnos de diferentes cursos; y la influencia de sus propios compañeros el uno del otro. Todo se discute en común y entusiasmo: lecturas, revelaciones artísticas, proyectos. Innumerables veces, por la tarde, saliendo de las clases van a la ciudad, en busca de una película, al final de la noche frente a un tranquilo milkshake, entonando las canciones antiguas francesas recientemente aprendidas" (Gilda e Souza apud Eva Blay, 2004: 68). 
Los días de estudiante de las pioneras se cuenta como una etapa especial, casi idílica, cuya sociabilidad sería muy diferente en relación a los años siguientes, en los que adquieren una vida profesional en la universidad. Es decir, además de los elogios dados a la buena parte del grupo docente de la facultad de los cursos en que se formaron, se establecen como representantes de los conocimientos, sino que también describen positivamente en sus respectivas clases ya que no era, como se describe Alice Canabrava, "oportunidad para la socialización entre los estudiantes de varias clases, con una animada conversación, cenas en la noche [...]" (Alice Canabrava apud Eva Blay, 2004: 93). Durante la graduación, las pioneras constatan que no había distinción en sus clases, es decir, los hombres y las mujeres fueron tratados de manera similar, sin diferencias, situación que cambió cuando empezaron a dar los primeros pasos en la vida profesional y académica, ya en este contexto, surgió un nuevo elemento en la escena: la competición.

Después de los primeros años de la carrera, las historiadoras reportan dos eventos que fueron significativos en sus caminos: la conquista de doctorado y la cátedra. Olga Pantaleão nos cuenta con tranquilidad algunas experiencias de su doctorado. Parece que enfrentó problemas importantes para hacerlo, teniendo la ayuda financiera recibida a través de una licencia de pago que le permitió ausentarse de la FFCL, ya que su investigación se realizó en la Biblioteca Nacional de Rio de Janeiro. Por otra parte, se dice que recibió ayudas para comprar los libros importados, con la mediación del Consejo Británico, y que eran necesarios para su investigación, la compra de la que fue autorizada por el Secretario de la USP. A Olga le fue concedido el doctorado en 1944, cuya tesis fue llamada La penetración en el mercado de la América española por la Inglaterra, 1713-1783. Por lo tanto, en un momento que hacer una tesis doctoral era una novedad, "comenzaron a aparecer las mujeres, participando en una tradición de investigación que dura hasta nuestros días." Por otra parte, describe las dificultades que tuvieron para las mujeres en relación con la defensa de la tesis:

"Durante la inscripción para el doctorado, las mujeres tenían las mismas facilidades que los hombres; pero tal vez en algunos casos tenían más dificultades en la defensa de la tesis, cuando era posible deshacerse del candidato indeseable. Sin embargo, el trabajo de preparación encontró a las instalaciones” (Olga Pantaleão apud Eva Blay, 2004: 115).

En estas dificultades, Alice Canabrava relata los acontecimientos más importantes. Al terminar su doctorado en 1942, cuya tesis recibió el título El comercio portugués en el Río de la Plata, la historiadora tentó el concurso para la cátedra de la Historia de América. Este proceso no produjo resultados positivos porque, dijo, hubo muchos obstáculos enfrentados. Algunos de ellos se relatan como sigue:

"Escuchar consejos y sugerencias que no se debe cuestionar la hostilidad jamás construida: sería fatalmente fallada. Mi respuesta era invariablemente la misma, sin ningún tipo de argumento: "Puedo hacer el concurso, serán ellos de la banca que me pueden reprobar. [...] Por lo tanto, cerrado y protegido por la organización interna del grupo, los colegas 
masculinos se utilizaban de un marco legal, y se prepararon para servir a sus intereses. Verificando la votación empatada, correspondía al presidente de la banca y la decisión final se toma en favor de mi competidor, bajo la justificación de que ya estaba en el cargo como profesor contratado" (Alice Canabrava, 2003: 20).

Pero el esfuerzo de Alice Canabrava no era inocuo. La Faculdade de Ciências Econômicas e Administrativas, de la USP, se organizó en 1946, y ella se trasladó allí. Se convirtió en la primera profesora catedrática de la Universidade de São Paulo en 1951, después de pruebas y títulos de competencia. De los profesores economistas siempre recibió “fuerte apoyo" para dedicarse a la Historia Económica del Brasil y entonces su historia se narra sin problemas cuando ella elige este tema, la transferencia para la FCEA como uno de los principales contextos favorables para la ascensión de su vida profesional.

De hecho, la ocupación de la cátedra era una de las principales dificultades para las mujeres que querían establecer sus carreras en cursos universitarios durante las primeras décadas desde la creación de la USP. A lo sumo, el máximo puesto que podría alcanzar era el de profesora titular. Como Alice Canabrava, Olga Pantaleón también utilizó la misma estrategia para posicionar y consolidar su profesión: celebró su traslado a la FFCL - Marília, que estaba en el inicio de sus actividades, y ejerció como Profesora Titular entre 1959-1975 y Directora de 1971 a 1975. De esta forma, Olga Pantaleão expone sus recuerdos sobre la cátedra, en la que su declaración se cruza con el testimonio de su colega profesional, Alice Canabrava:

"Por último, el Presidente, puesto final en la carrera universitaria en ese momento, estaba fuera del alcance de las mujeres: se levantó un baluarte masculino contra la mera posibilidad. Una mujer hizo que la competencia para la Historia de la Civilización Americana Presidente: Alice Piffer Canabrava. ¡Fue un evento! Pero, a pesar de haber obtenido las calificaciones más altas, no ganó el concurso: la junta de examen eligió el segundo de notas, hombre, por supuesto, dándole la Cátedra; este disparate era posible, entonces, en virtud de las normas de competencia, lo que permitió al banco a votar por el candidato que quiera ser el primero. Eso era todo lo que ocurrió en el 40, en referencia a la ocupación de la Cátedra de las mujeres” (Olga Pantaleão apud Eva Blay, 2004: 113).

\section{Consideraciones finales}

En el libro Mulheres Públicas, la historiadora Michelle Perrot escribió “incluso más que el espacio material es la palabra y su circulación que dan forma a la esfera pública" (Michelle Perrot, 1998: 59). La idea de que la naturaleza de las mujeres está destinada al silencio y la oscuridad era hasta hace poco profundamente arraigada en nuestras culturas. En Brasil, en las primeras décadas del siglo XX, las mujeres se limitaban absolutamente al espacio privado, 
permaneciendo durante mucho tiempo excluidas del espacio público y político. Sin embargo, podemos decir que uno de los factores que diseñaron las mujeres brasileñas en el espacio público fue su entrada en la universidad. A través del acceso a la universidad las mujeres se posicionaron en el mundo de la escritura, de la voz y, por lo tanto, de la vida pública, logrando pasar de la invisibilidad a la visibilidad.

Los testimonios de las seis pioneras revelan facetas de una sociedad machista y patriarcal que limitaba las acciones de las mujeres en la esfera pública. En términos profesionales, había pocas oportunidades para desarrollar una carrera que no se limitase en "cuidar a los demás" como maestra, enfermera o trabajador social. Sin embargo, el acceso a la educación superior y la dedicación a la carrera universitaria cambió gradualmente las relaciones de sociabilidad entre hombres y mujeres en la esfera pública. En esta perspectiva, el trabajo intelectual y la enseñanza en la universidad, en su historicidad, son actividades de la vida relacionada con un espacio social, el tiempo y el desempeño de las agentes examinadas.

En los testimonios analizados, podemos entender la profesión de las académicas no sólo como un factor de generación de ingresos propios, sino también como un medio para el desarrollo personal, con impacto en la autoestima y la posibilidad de ocupación de espacios publicos y por lo tanto, ganar el reconocimiento en ambientes que antes no fueran sido ocupados por mujeres. Para reflejar este problema, aquí traemos el pensamiento de Claude Dubar que defiende la tesis de la centralidad del trabajo en la vida personal y el lugar eminente de identificación profesional en la vida social, en la que el hecho de ser reconocido en su trabajo, establecer relaciones, incluso conflictos con los demás y para ser capaces de participar personalmente en su actividad es, al mismo tiempo, constructor de la identidad personal y la inventiva social (Claude Dubar, 2005). En el caso de las pioneras, esto se refleja en la forma en que eligieron para expresar sus carreras profesionales, es decir, sus discursos están marcados por una satisfacción y el reconocimiento de su propio valor en la constitución del campo disciplinar en la que actuaron.

Los informes también revelan que sus trayectorias profesionales las llevaron a caminos diferentes y, a veces, lejos de la investigación y la enseñanza. La multiplicidad del campo de trabajo dentro y fuera de las universidades es instructiva en el sentido de cómo el prestigio y el reconocimiento se pueden construir de otra manera y en otros círculos que van más allá del modelo restrictivo de la productividad académica. Muchos, si no la mayoría, ocuparon puestos de dirección y coordinación de los departamentos, asociaciones, editoriales y en Ayuntamientos, cuyas actividades también han configurado la capacitación de estas mujeres.

Por una parte, centramos nuestro análisis en lo que se dijo, por otro lado, también debemos reflexionar sobre lo que no se ha dicho, es decir, sobre cuestiones que todavía plantean conflictos y tensiones para las mujeres que trabajan, independientemente de las actividades profesionales comprometidas. Se trata de reconocer la relación entre la vida profesional y la vida familiar, es decir, la tensión entre la esfera pública y privada. En un testimonio estudiado ninguna indicación en cuanto a cómo las pioneras tratarán aspectos conducentes al matrimonio, 
los hijos, la vida personal y familiar. Uno se pregunta: ¿las que estaban casadas, tenían el apoyo y el reconocimiento de sus maridos? ¿Cuáles fueron las dificultades que enfrentaron en la división de las funciones sociales de la pareja? ¿La maternidad era un impedimento para el ejercicio de algunas actividades o puestos de trabajo? ¿No se pudo realizar un postgrado en otro país debido a la familia? ¿Cuáles fueron las negociaciones en la familia para que pudieran desarrollar sus carreras universitarias? ¿Algunas de ellas abandonó la vida amorosa y reproductiva en detrimento de la vida laboral? De todos modos, estos fueron los temas que no se problematizaron en sus discursos.

Esto nos deja dos reflexiones importantes. Por un lado, que se tiene una visión permanente que el trabajo del profesor universitario, investigador y sobre todo el desarrollo intelectual, es algo indisociable de toda actividad relacionada con la vida doméstica y personal. En este sentido, el espacio universitario y el trabajo intelectual se tratan como terrenos neutrales, donde no hay interferencia de discursos, prácticas y experiencias de la vida personal, social, económica y cultural de los agentes que actúan sobre ellos. En segundo lugar, se sabe que la entrada de la mujer en la vida pública, tanto desde su incorporación en el mercado laboral y en la escolarización hay avances visibles y que la participación política, no fue acompañada por la inserción del hombre en la responsabilidad con las tareas reproductivas, históricamente femenina. Con lo cual, en consecuencia, se argumenta que la inclusión/acceso no es suficiente para afirmar el orden de los procesos de dominación, explotación, característica del orden patriarcal, especialmente en un área ocupacional donde la alta cualificación, la formación continua, la producción científica, circulación nacional e internacional son inherentes a la progresión y la promoción profesional y el acceso a mejores salarios. Por lo tanto, estas dos cuestiones están siendo tratadas con mayor fuerza sólo en los últimos años, a través de los estudios feministas, que en la década de 1980 era incuestionable.

Por último, el testimonio aquí analizado desde la perspectiva de expresión nos ayuda a pensar cómo las jóvenes estudiantes de la década de 1930 se dieron cuenta de que podían construir sus vidas más allá de los límites establecidos para las mujeres. Estudiaran, se formaron y muchas de ellas han desarrollado brillantes carreras universitarias. Posiblemente han enfrentado dificultades de conciliación entre el matrimonio y la crianza con la vida intelectual, el trabajo remunerado y la igualdad de género. Introducirse en estas nuevas actividades fue, y sigue siéndolo hoy en día, muy costoso. La ruptura de modelos tradicionales o la posibilidad de modelos alternativos de vida y subjetividades, no encuentran apoyo en la sociedad que busca preservar su forma de vida ordenado por la sumisión de la mujer y al mismo tiempo no ofrece alternativas a las profesionales, tales como guarderías en lugares a donde trabajan. Ellas tuvieron que superar la ideología patriarcal en la que fueron educadas; tratar de abrir nuevas áreas de apoyo que les permitieron incorporarse en nuevas profesiones y crear un espacio de visibilidad, lo que incluye el "poder de la palabra y la escritura", que les garantizaría un lugar entre sus colegas varones de profesión. 


\section{BibLIOGRAFÍA}

- Arfuch, Leonor (2010): O espaço biográfico: dilemas da subjetividade contemporânea. Rio de Janeiro: EdUERJ.

- Barros, Diana L. P. y Fiorin, José (1999): Dialogismo, polifonia, intertextualidade. São Paulo, SP: Edusp.

- Blay, Eva Alterman y Lang, Alice Beatriz da Silva Gordo (1984): “A mulher nos primeiros tempos da Universidade de São Paulo". En: Ciência e Cultura. São Paulo vol. 36, nº 12, dezembro, pp. 2135-2143. (2004): Mulheres na USP: horizontes que se abrem. São Paulo: Associação Editorial Humanitas.

- Canabrava, Alice P. (2003): O caminho percorrido. São Paulo: Associação Brasileira de Pesquisadores em História Econômic.

- Dubar, Claude (2005): A Socialização: construção das identidades sociais e profissionais. São Paulo: Martins Fontes.

- Foucault, Michel (1998): A ordem do Discurso. São Paulo: Ed. Loyola.

- Hall, Beatriz (2010): "La construcción de sentido: el caso de los enunciados metafóricos y el discurso académico". En: Revista Semántica e interpretación. Tópicos del Seminario, 23, enero-junio, pp. 191-220. Versión [en línea] Disponible en: http://www.scielo.org.mx/pdf/tods/n23/n23a6.pdf [02/01/2016].

- Liblik, Carmem Silvia da Fonseca Kummer (2014): "A participação das mulheres na construção do conhecimento histórico”. En: Revista Feminismos, vol. 2, no. 3, Set.-Dez., pp. 55-68.

(2015): “A formação e a profissionalização de professoras universitárias brasileiras (1960-1980). En: História Oral, vol. 18, nº. 2, jul./dez., p. 7-34.

- Lima, Nadia Regina Loureira de Barros (2002): "As mulheres nas ciências: o desafio de uma passagem... a passagem do privado para o público". En: Ana lice A. Costa y Cecília Maria Sardenberg: Feminismo, Ciencia e Tecnologia. Salvador: REDOR/NEIM-FFCH/UFBA, pp. 51-65.

- Maingueneau, Dominique (1989): Novas tendências em análise do discurso. Campinas: Martins Fontes.

- Orlandi, Eni (1983): A linguagem e seu funcionamento. As formas do discurso. São Paulo: Editora Brasiliense.

- Pantaleão, Olga (2004): "Olga Pantaleão. Historiadora". En: Eva Alterman Blay y Alice Beatriz da Silva Gordo Lang: Mulheres na USP: horizontes que se abrem. São Paulo: Associação Editorial Humanitas, pp. 107-115.

- Pêcheux, Michel (2002): O Discurso: estrutura ou acontecimento. 3a ed. Campinas (SP): Pontes.

- Perosa, Graziela (2005): "Educação diferenciada e trajetórias profissionais femininas". En: Tempo Social. Revista de Sociologia da USP, vol. 20, nº. 1, pp. 51-68.

- Perrot, Michelle (1998): Mulheres Públicas. São Paulo: UNESP.

- Santos, Silmere Alves (2015): Trabalho docente, família e vida pessoal. Permanências, deslocamentos e mudanças contemporâneas. São Paulo: Paco Editorial.

- Schimidt, Benito (2000): "Luz e papel, realidade e imaginação: as biografias na história, no jornalismo, na literatura e no cinema". En: Benito Schmidt (org.): O biográfico: perspectivas interdisciplinares. Santa Cruz do Sul: UNISC, pp. 49-70.

- Tiburi, Márcia (2002): As Mulheres e a Filosofia. São Leopoldo: Ed. Unisinos.

- Trigo, Maria Helena B. (1997): "Espaços e tempos vividos: estudo sobre os códigos de sociabilidade e relações de gênero na Faculdade de Filosofia da USP (1934-1970)". São Paulo. Tese de Doutorado Universidade de São Paulo. 